\title{
Uso de anticoagulantes em pacientes hospitalizados por trombose venosa profunda
}

\section{em membros inferiores}

\author{
Use of anticoagulants in patients hospitalized for deep vein thrombosis in the lower limbs \\ Uso de anticoagulantes en pacientes hospitalizados por trombosis venosa profunda en miembros
}

inferiores

Recebido: 02/11/2021 | Revisado: 09/11/2021 | Aceito: 10/11/2021 | Publicado: 16/11/2021

Patrícia Lima Gomes

ORCID: https://orcid.org/0000-0003-0708-7471 Universidade Nilton Lins, Brasil E-mail: patricia.lima04245@gmail.com Adrielle Aquino da Silva ORCID: https://orcid.org/0000-0002-9572-4946 Universidade Nilton Lins, Brasil

E-mail: aquinoadrielle@gmail.com

Omero Martins Rodrigues Junior

ORCID: https://orcid.org/0000-0002-8552-3278 Universidade Nilton Lins, Brasil

E-mail: omeromartins.farm@gmail.com

\begin{abstract}
Resumo
A trombose venosa profunda (TVP) é definida como um evento trombótico que envolve as veias profundas dos membros inferiores, veias poplíteas, femurais ou ilíacas. A TVP geralmente é acompanhada por embolia pulmonar (EP) e o Tromboembolismo venoso (TEV) é um termo usado para especificar as duas possibilidades. Desse modo, esta pesquisa teve como objetivo a realização de uma revisão bibliográfica sobre o uso dos anticoagulantes em pacientes hospitalizados e a atuação do farmacêutico na orientação da equipe multidisciplinar hospitalar sobre a utilização adequada dos fármacos. Trata-se de uma revisão bibliográfica por meio de uma abordagem qualitativa, através de fontes secundárias para descrição sobre o uso de anticoagulantes em pacientes com trombose venosa profunda em membros inferiores. Por meio dos resultados obtidos verifica-se que, cabe ao farmacêutico instruir o paciente quanto à utilização do medicamento, dose, melhor horário de administração, interação medicamentosa, além de hábitos saudáveis no cuidado não terapêutico, podendo fazer intervenções que vão desde a simples sinalização de uma interação, a sugestões de terapias alternativas ou ajustes nas doses prescritas pelo médico, quando identificado algum erro ou problemas relacionados à terapia. Esse ajuste deve ser documentado e informado ao médico prescritor, constituindo um elo entre farmacêutico, médico e paciente.
\end{abstract}

Palavras-chave: Trombose Venosa Profunda; Membros inferiores; Anticoagulantes; Profissional farmacêutico.

\begin{abstract}
Deep vein thrombosis (DVT) is defined as a thrombotic event involving the deep veins of the lower limbs, popliteal, femoral or iliac veins. DVT is usually accompanied by pulmonary embolism (PE) and venous thromboembolism (VTE) is a term used to specify both possibilities. Thus, this research aimed to carry out a literature review on the use of anticoagulants in hospitalized patients and the role of the pharmacist in guiding the multidisciplinary hospital team on the proper use of drugs. This is a literature review using a qualitative approach, using secondary sources to describe the use of anticoagulants in patients with deep vein thrombosis in the lower limbs. Through the results obtained, it appears that it is up to the pharmacist to instruct the patient about the use of the drug, dose, best time of administration, drug interaction, in addition to healthy habits in non-therapeutic care, being able to make interventions that range from simple signaling from an interaction, to suggestions for alternative therapies or adjustments to the doses prescribed by the physician, when any error or problems related to the therapy are identified. This adjustment must be documented and informed to the prescribing physician, constituting a link between pharmacist, physician and patient.
\end{abstract}

Keywords: Deep Vein Thrombosis; Lower members; Anticoagulants; Pharmacist professional.

\section{Resumen}

La trombosis venosa profunda (TVP) se define como un evento trombótico que involucra las venas profundas de los miembros inferiores, venas poplíteas, femorales o ilíacas. La TVP suele ir acompañada de embolia pulmonar (EP) y tromboembolismo venoso (TEV) es un término utilizado para especificar ambas posibilidades. Así, esta investigación 
tuvo como objetivo realizar una revisión de la literatura sobre el uso de anticoagulantes en pacientes hospitalizados y el papel del farmacéutico en la orientación del equipo multidisciplinario del hospital sobre el uso adecuado de los fármacos. Se trata de una revisión de la literatura con abordaje cualitativo, utilizando fuentes secundarias para describir el uso de anticoagulantes en pacientes con trombosis venosa profunda en miembros inferiores. A través de los resultados obtenidos, parece que le corresponde al farmacéutico instruir al paciente sobre el uso del fármaco, dosis, mejor momento de administración, interacción farmacológica, además de hábitos saludables en los cuidados no terapéuticos, pudiendo realizar intervenciones que van desde la simple señalización de una interacción, hasta sugerencias de terapias alternativas o ajustes a las dosis prescritas por el médico, cuando se identifica algún error o problema relacionado con la terapia. Este ajuste debe documentarse e informarse al médico que prescribe, constituyendo un vínculo entre el farmacéutico, el médico y el paciente.

Palabras clave: Trombosis Venosa Profunda; Miembros inferiores; Anticoagulantes; Profesional farmacéutico.

\section{Introdução}

A Trombose Venosa (TV) ocorre por intermédio de formações de coágulos sanguíneos nas veias do corpo, bloqueando o fluxo sanguíneo, causando edemas e dores na região que foi afetada, na maioria das vezes acometem as partes inferiores, como as panturrilhas e coxas, mas podem ocorrer também nos membros superiores (Brasil, 2020a; Brasil, 2020b). Entre a incidência de TV, a TVP é potencialmente mais grave, pois os trombos atingem artérias pulmonares, causando EP (Shen et al., 2017). Tendo a formação de trombos explicada através de mecanismos fisiopatológicos (Wolberg et al., 2011) que é desencadeado por fatores como: alterações do fluxo sanguíneo, lesão endotelial vascular e estado de hipercoagulabilidade, sendo esses fatores predispostos pela teoria da tríade de Virchow (Mioc et al., 2018).

Na concepção de Sousa \& Álvares (2018), os fatores de riscos para TVP são classificados de forma separada de três formas, sendo a primeira por características hereditárias (Resistência a proteína $\mathrm{S}$ ativada, mutação da protrombina, deficiência da proteína C e S, aumento do fator III e diminuição do fibrinogênio), seguindo por características adquiridas (Câncer, Idade, Obesidade, Trauma, cirurgias e imobilização) e por associações prováveis (Aumento de lipoproteína A, baixos níveis de inibição do fator tecidual, níveis aumentados de fibrinogênio). A presença de pelo menos um dos fatores de risco está associado ao desenvolvimento de TVP entre os pacientes hospitalizados, tanto pacientes clínicos, como pacientes cirúrgicos (Farhat, Gregório \& Carvalho, 2018). Entre os fatores de riscos mais encontrados em pacientes clínicos hospitalizados, são: obesidade, doenças respiratórias agudas graves e insuficiência cardíaca congestiva. Já nos cirúrgicos, a classificação de riscos depende do tipo de procedimento cirúrgico a qual o paciente foi submetido (Fuzinatto et al., 2011).

A terapia medicamentosa dessa condição é a base de anticoagulantes para prevenir do avanço para TV e síndrome pós-trombótica para o tratamento e redução de recorrência do TV. Sendo a terapia tradicional inicial realizada com Heparina não fracionada (HNF) ou Heparina de baixo peso molecular (HBPM), o tratamento com as heparinas tem duração de 5 a 7 dias, e em seguida é utilizado por longo prazo a Varfarina, que é um antagonista da Vitamina K (Yoshida, 2016; Brandão et al., 2018). O principal efeito adverso relacionado a terapia medicamentosa e que é presente em ambas as medicações, é a hemorragia. Sendo que os pacientes em uso de Varfarina e que possuam idade avançada, doença renal crônica, disfunção hepática e câncer, o risco do efeito adverso é aumentado. Já no uso das heparinas, a incidência de hemorragia pode variar de acordo com a posologia e via de administração (Harter, Levine \& Henderson, 2015; Raphael, 2020).

A TV tem prevalência mundial de 50 a 200 casos por 100 mil habitantes/ano (Ohki \& Van Bellen, 2017). Pacientes com Covid-19 podem desenvolver TV e consequentemente EP. Durante o pico da pandemia, estudos apontam que houve um aumento exacerbado de TV no mundo. No Reino Unido, de 274 pacientes hospitalizados, 76,2\% foram diagnosticados com EP e 23,8\% com diagnóstico de TVP (Brasil, 2020c). A ocorrência de TVP em pacientes hospitalizados no Brasil é pouco explorada e instável na literatura (Okuhara et al., 2015).

No Brasil, o tromboembolismo venoso (TEV) pode iniciar-se por volta dos 30 anos, nos Estados Unidos e na Europa começa aos 40 anos, porém a ocorrência mais frequente dessa patologia é em pacientes com 60 e 70 anos. O tromboembolismo 
pulmonar está intimamente relacionado a tumores malignos e todos os fatores que aumentam a estase de coagulação ou dano endotelial vascular aumentam a possibilidade de trombose e tromboembolismo (Alves, Almeida \& Balhau, 2015). Calcula-se que cerca de 300.000 habitantes dos Estados Unidos da América (EUA) e na Europa mais 500 mil apresentam TVP e TEP todos os anos. O Brasil não possui um registro preciso da incidência, mas considera-se que a taxa de trombose seja de 1 ou 2/100 residentes. Dentre os fatores de risco, destacam-se: genética e meio ambiente, tabagismo, idade, sexo, cirurgia plástica, gravidez e pós-parto, contracepção e reposição hormonal (Souza \& Álvares, 2018).

A segurança das prescrições e a confiança no manejo de suas complicações, especialmente complicações hemorrágicas, reforçam o caráter multiprofissional envolvido na prevenção do TEV, havendo a necessidade de aprimoramento da segurança do paciente já nas primeiras horas de internação.

De acordo com todo o contexto já exposto, leva-se em consideração que o tratamento principal consiste no uso de anticoagulantes visando basicamente o controle de recorrência da TV e consequentemente da EP. Se torna imprescindível a atuação do farmacêutico junto a equipe multidisciplinar para que o tratamento seja feito de forma adequada para cada tipo de situação com o objetivo mínimo de efeitos adversos entre as terapias medicamentosas. Se faz necessário o acompanhamento completo de profissionais capacitados, incluindo farmacêuticos clínicos, a fim de promover o serviço de anticoagulação de forma regular e contínuo, fazendo o gerenciamento dos efeitos adversos, interações, ajuste de doses, e em casos de inadequação o farmacêutico deve realizar uma revisão do quadro medicamento junto a equipe multidisciplinar.

Desse modo, esta pesquisa tem como objetivo realizar uma revisão bibliográfica sobre o uso dos anticoagulantes em pacientes hospitalizados e a atuação do farmacêutico na orientação da equipe multidisciplinar hospitalar sobre a utilização adequada desses fármacos e tem como objetivos específicos: descrever o mecanismo de ação dos anticoagulantes, identificar os efeitos adversos, as contraindicações do fármaco e descrever o papel do farmacêutico na orientação da equipe multidisciplinar.

\section{Metodologia}

Trata-se de uma revisão bibliográfica, por meio de uma abordagem qualitativa, utilizando-se de fontes secundárias para chegar aos objetivos expostos, onde foram utilizados para pesquisas as plataformas Scientific Electronic Library Online SCIELO, Medical Literature Analysis and Retrievel System Online - MEDLINE/PUBMED, Google Acadêmico e Ministério da Saúde. Utili de forma associada os descritores: "Trombose Venosa", "Trombose Venosa Profunda", "Membros inferiores", "Anticoagulantes" e "Profissional Farmacêutico", nos idiomas: português, inglês e espanhol. Os estudos foram obtidos nos meses de agosto a outubro de 2021, onde foram contemplados artigos, legislações e dissertações nas plataformas já mencionadas, dentro do tempo estipulado de dez anos (2011 a 2021).

Os critérios de exclusão se deram por data fora do período estipulado, sem a temática abordada e estudos repetidos nas bases de dados. Para os critérios de inclusão, foram utilizados artigos em coerência com o tema disposto, publicados dentro do tempo estabelecido, estudos completos e de acesso livre. Após aplicação de critérios de exclusão e inclusão, deu-se seguimento a leitura dos estudos na integra para estudos potencialmente elegíveis. Ao final foram obtidos 36 estudos.

\section{Resultados e Discussão}

\subsection{Fisiopatologia}

A fisiopatologia está inteiramente ligada a TVP, pois aproximadamente 79\% dos pacientes com EP possuem TVP nos membros inferiores e 50\% dos pacientes com TVP evoluem para EP. No Brasil em 2014 foram 122.096 internações por TEV e em 2015 foram registrados 113.817, havendo uma queda neste ano de 8.279 internações (Brasil, 2016). Na EP, as alterações do 
parênquima pulmonar são mínimas, às vezes encontra-se atelectasia, sinais de oclusão do vaso comprometido (hipertransparência, infarto pulmonar, entre outros) e derrame pleural, que é mais comum se houver correlação com a insuficiência cardíaca; o infarto pulmonar é encontrado de 5\% a 15\% (Pereira, 2011).

As causas mais comuns de TVP nas extremidades inferiores são o retorno venoso prejudicado em pacientes imobilizados, lesão após fratura da perna ou disfunção endotelial e hipercoagulabilidade causada por cateteres venosos centrais, marca-passos ou drogas injetáveis.

Foram definidos três fatores predisponentes, chamada de "tríade de Virchow", que é composta pelo estado de hipercoagulabilidade, estase venosa e lesão da parede endotelial, conforme a faixa etária de que consiste na principal causa de TEV. A hipercoagulação é uma adaptação do organismo materno, entre as idades de 20 a 40 anos a maior incidência ocorre em mulheres por estarem em idade fértil e por estarem expostas aos fatores de riscos como uso de anticoncepcionais e gestação (Sousa \& Àlvares, 2018).

Com isso, desde a concepção, iniciam-se as alterações na cascata de coagulação, como a diminuição das proteínas anticoagulantes C e S e o aumento de fibrinogênio e dos fatores V, VIII, IX e X. A estase venosa nas extremidades inferiores ocorre pela compressão uterina, juntamente com a vasodilatação induzida pela progesterona. Por fim, a lesão endotelial acontece devido ao parto, principalmente, em complicações que resultam em um trauma cirúrgico (Mendonça et al., 2021).

\subsection{Hemostasia}

A hemostasia é a interrupção de sangue dos vasos sanguíneos danificados sendo essencial à sobrevivência. Os principais fenômenos são adesão, ativação plaquetária e a formação de fibrina. A hemostasia do sangue é determinada por uma série de processos em que o sangue muda de um estado líquido para um estado mais viscoso, semelhante a um gel, para formar um coágulo. Essa transição é para atingir a estabilidade e a homeostase, que antes eram perdidas devido à ruptura dos vasos sanguíneos e perda de sangue, evitando assim a perda maciça (Rang et al., 2016).

O mecanismo de hemostasia inclui três processos: hemostasia primária, hemostasia secundária (onde ocorre a coagulação) e a fibrinólise. Todos esses processos têm um propósito de manter a fluidez do sangue necessária para evitar o extravasamento de vasos sanguíneos ou bloquear o fluxo quando o trombo está presente (Cagnolati et al., 2017).

\subsubsection{Hemostasia Primária}

A hemostasia primária é responsável pela hemostasia por vasoconstrição e formação de trombo ou coágulos plaquetários (Zago et al, 2013). As plaquetas entram em contato e se aderem aos vasos sanguíneos danificados, como fibras de colágeno na cartilagem hialina abaixo das células endoteliais danificadas. Este processo é denominado adesão plaquetária, pois é nele que se forma o tampão plaquetário. Após o resultado da adesão, as plaquetas são ativadas e suas propriedades sofrem alterações fundamentais. As plaquetas emitem muitos projéteis, permitindo-lhes entrar em contato e interagir com outras plaquetas e começar a liberar o conteúdo de suas vesículas. Este estágio é chamado de resposta de liberação de plaquetas. A liberação de Adenosina difosfato (ADP) torna outras plaquetas nas áreas pegajosas, e a viscosidade das plaquetas recémrecrutadas e ativadas faz com que essas plaquetas grudem nas plaquetas originalmente ativadas, este processo é agregação plaquetária. O acúmulo e a adesão de um grande número de plaquetas formam uma substância denominada embolia plaquetária (Silva \& Melo, 2016).

\subsubsection{Hemostasia secundária}

A hemostasia secundária é também conhecida como um sistema de coagulação, pois envolve a conversão de proteínas solúveis do sangue, plasma, fibrinogênio, no polímero insolúvel fibrina por meio da ação de enzimas chamado de trombina. A 
fibrina forma uma rede de fibras elásticas fortalecendo as plaquetas que obstruem e transformam o tampão hemostático (Cagnolati et al., 2017).

A coagulação depende de várias substâncias chamadas fatores de coagulação. Esses fatores incluem íons de cálcio, várias enzimas inativas sintetizadas pelas células do fígado e liberadas na corrente sanguínea e várias moléculas relacionadas às plaquetas ou liberados pelos tecidos lesados. Após esta liberação os fatores de coagulação são identificados por números romanos que identificam a sua ordem de participação do processo de coagulação. Essa coagulação sanguínea envolve um sistema de amplificação, no qual poucos materiais iniciais são ativados sequencialmente, por meio de proteólise, cascatas de proteínas precursoras circulantes, fatores enzimáticos de coagulação e produção de trombina, este transforma o fibrinogênio plasmático solúvel em fibrina. A fibrina penetra nos agregados de plaquetas no local da lesão vascular transformando em tampões hemostáticos fortes e estáveis (Silva \& Melo, 2016).

\subsubsection{Fibrinólise}

A fibrinólise refere-se ao processo de digestão por proteases específicas da fibrina. No estanto, o sistema fibrinolítico é semelhante ao sistema de coagulação, pois a plasmina forma precursora da serinaproteases, circula de forma inativa como o plasminogênio. Em resposta à lesão, as células endoteliais sintetizam e liberam ativadores. O plasminogênio tecidual (t-PA) se converte ao plasminogênio em plasmina. A plasmina remodela o trombo e limita sua expansão por meio da digestão proteolítica da fibrina. Tanto o plasminogênio quanto a fibrina têm domínios proteicos (kringles), que se ligam à lisina exposto no coágulo de fibrina para fornecer especificidade para o processo de fibrinólise do coágulo. No nível farmacológico do t-PA usado na terapia trombolítica, a especificidade dos coágulos sanguíneos é perdida, ocorrendo o estado de dissolução sistêmica e o aumento do risco de sangramento (Brunton, Hilal-Danda \& Knollmann, 2018).

A fibrinólise é regulada principalmente pela ação do Inibidor do Ativador de Plasminogênio-1 (PAI-1), a $\alpha_{2}$ antiplasmina ( $\alpha 2$-AP) e Inibidor Frinolítico Ativado pela Trombina (TAFI). O PAI-1 é produzido pelo endotélio vascular e plaquetas e inibe o t-PA e Ativador de Plasminogênio Urocinase (u-PA). A $\alpha 2$-AP inibe a ação da plasmina. Na presença de Trombomodulina (TM), a TAFI ativada pela trombina, inibe a fibrinólise por meio da modificação do substrato de Fibrina. Portanto, a TAFIa elimina o resíduo C- terminal. A remoção de arginina e lisina da fibrina são degradadas, resultando na redução da ligação e ativação de Pg de superfície de fibrina (Zago, et al., 2013).

\subsubsection{Cascata de coagulação}

A coagulação é causada por um dano tecidual que representa o processo básico de homeostase e manutenção da integridade dos vasos sanguíneos. Embora seja essencial para combater a perda excessiva de sangue, ela precisa ser regulamentada para evitar a formação de trombos devido à produção excessiva de fibrina (Rodrigues et al., 2012). A análise avaliativa do papel das células nas etapas de hemostasia pode construir um modelo de coagulação que explica melhor o sangramento e a trombose in vivo. Logo, ela depende de várias substâncias chamadas fatores de coagulação. Esses fatores incluem íons de cálcio, várias enzimas inativas, sintetizadas pelas células do fígado. As células do fígado são liberadas no sangue, bem como várias moléculas relacionadas às plaquetas. Muitos fatores de coagulação são identificados por números romanos (Quadro 1), não necessariamente seguindo a ordem em que participaram durante o processo de solidificação (Silva \& Melo, 2016). 
Quadro 1. Fatores de Coagulação Sanguínea.

\begin{tabular}{|c|c|}
\hline Fator & Sinônimo \\
\hline I & Fibrinogênio \\
\hline II & Protombina \\
\hline III & Fator tecidual ou Tromboplastina Tecidual \\
\hline IV & Cálcio \\
\hline V & Proacelerina \\
\hline VII & Proconvertina \\
\hline VIII & Fator de vonWillebrand (FvW) \\
\hline IX & Fator de Christmas, componete tromboplastínico plasmático (PCT) \\
\hline X & Antecedente tromboplastínico plasmático (PTA) \\
\hline XI & Fator estabilizador da fribina \\
\hline XII & XIII
\end{tabular}

Fonte: Adaptado de Katzung, Masters e Trevor (2014).

O fator tecidual é o receptor do fator VII que na presença do $\mathbf{C a}^{2+}$, se transforma em ativo, levando à ativação catalítica do fator VII em VIIa. O complexo do fator VIIa do tecido ativa o cofator IX e X. As plaquetas também funcionam secretando fatores de coagulação, como o fator Va e o fibrinogênio. O complexo IXa - VIIIa - $\mathbf{C a}^{2+}{ }^{+}$-fosfolipídeo produz mais fator Xa para apoiar a coagulação do sangue. Se faz necessário, pois o complexo do fator VIIa é rapidamente inativado no plasma pelos inibidores que levam as células e pela antitrombina III. $\mathrm{O}$ fator Xa ativa a protrombina em trombina na presença de $\mathbf{C a}^{2+}$, fosfolipídios e fator Va, que é a principal enzima da reação em cascata. Quando o fator XII (fator de Hageman) se adere à superfície com carga elétrica negativa e se funde com a via de fase de ativação do fator X, começa a via intrínseca. O fator IIa cliva o fibrinogênio para produzir fragmentos que polimerizam para formar fibrina, ativando o fator XIII. A fibrinoligase fortalece a ligação fibrina-fibrina para estabilizar o coágulo. (Rang et al., 2016). Como demonstrado na Figura 1. 
Figura 1. Cascata de coagulação.

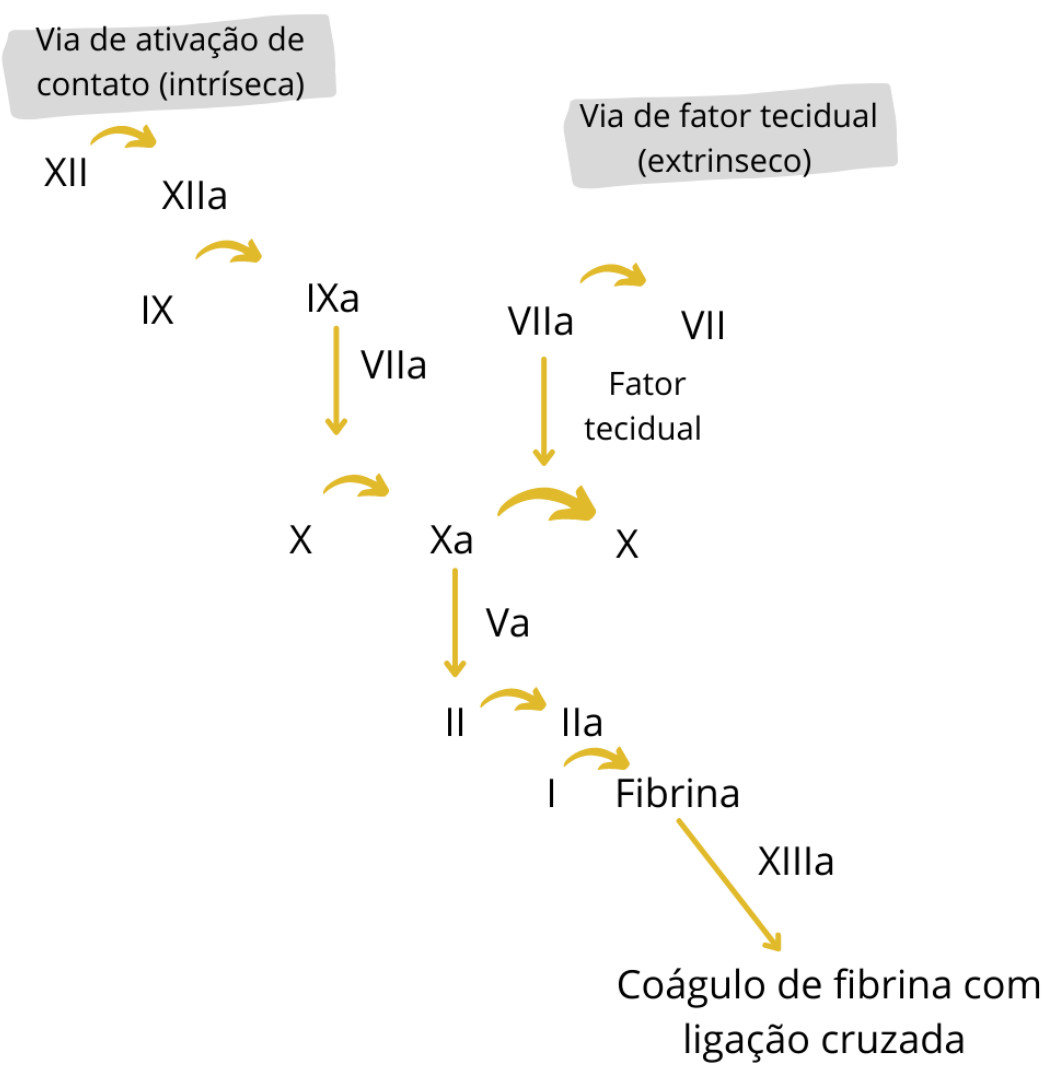

Fonte: Adaptado de Harter, Levine e Henderson (2015).

Um dos exames laboratoriais usados para monitorar o tratamento é o Tempo de Protombina, avaliando as vias externas e comuns de coagulação, dependendo da integridade dos fatores VII, V, II e X. Quando a tromboplastina e o cálcio tecidual são adicionados, o teste mede o tempo que o sangue leva para formar um coágulo de fibrina em segundos (Zago et al., 2013).

\subsection{Mecanismo de ação dos Anticoagulantes}

Os anticoagulantes são utilizados como base terapêutica para pacientes com a TV, o tratamento pode ser dividido em três fases: a primeira sendo a fase aguda (primeiros 5-10 dias) sendo utilizados para terapia anticoagulantes de uso parenteral com início rápido as HNF ou HBPM; a segunda é a fase de longo prazo (primeiros 3 meses), sendo a terceira de prolongado (acima de 3 meses) tempo de terapia. Geralmente para a segunda e terceira fase são utilizados agentes anticoagulantes orais, que se destaca o uso da Varfarina para ambos casos (Nutescu et al., 2016).

As heparinas são administradas por via endovenosa (EV) ou subcutânea (SC), devido não haver absorção no intestino, quando administrada por via oral, devido a sua carga molecular. Por via EV a heparina age imediatamente, porém na administração por via SC sua ação inicia em até 60 minutos, sendo assim em situações em que seja necessário um efeito imediato, a via de utilização deve ser a EV (Zago et al., 2013; Katzung, Masters \& Trevor, 2014).

As heparinas atuam inibindo a coagulação, através da ativação da antitrombina III, que inibe a trombina e outras serina-proteases por ligação ao sitio ativo, porém para essa inibição é necessário que a heparina se ligue tanto a enzima como a antitrombina III. Já para inibição do fator Xa, é fundamental que a heparina se ligue somente a antitrombina III, sendo assim as HBPM, como é o caso da Enoxaparina, atuam se ligando a antitrombina III, aumentando assim sua ação sobre o fator Xa. As HBPM não conseguem ativação da ação da antitrombina III sobre a trombina, pois possuem moléculas pequenas, não 
conseguindo se ligar a enzima e a trombina simultaneamente, esse processo é essencial para inibir a trombina, porém não para inibir o fator Xa (Rang et al., 2016).

A HNF também consegue se ligar à antitrombina, e devido a sua cadeia ser maior, formam o complexo HNF/AT, esse por sua vez é responsável pela inativação da trombina ou fator IIa e respectivamente dos fatores Xa, IXa, XIa e XIIa, sendo este a constituição do principal mecanismo anticoagulante da HNF. Porém a HNF pode se ligar ao cofator II e inativar a trombina, sem depender necessariamente da presença de antitrombina, mas para que isso ocorra são necessárias concentrações maiores de HNF no plasma (Zago et al., 2013).

Por outros lado, os antagonistas de vitamina k, também denominados como anticoagulantes cumarínicos, que tem como representante principal a varfarina, são anticoagulantes orais utilizados para prevenção e tratamento da TV e eventos tromboembólicos, apresentam efeitos anticoagulantes pois atuam bloqueando a transformação da vitamina $\mathrm{k}$ oxidada em vitamina $\mathrm{k}$ reduzida, sendo essa a forma atuante como uma coenzima na gama-carboxilação e interferem no processo biológico, tornando inativos, ou seja, até são produzidas no fígado, porém perdem a capacidade de serem ativadas, bloqueando assim o processo dos fatores dependentes de vitamina $\mathrm{k}$, como os fatores II, VII, IX e X. Os fatores que regulam a coagulação sofrem a mesma influência, sendo elas as proteínas C, S e Z, que não são capazes de serem ativadas (Katzung, Masters \& Trevor, 2014; Patel et al., 2021).

Portando os efeitos dos antagonistas de vitamina K serão efetivadas de acordo com a substituição de fatores ativos para fatores inativos. Dependendo também da meia vida de cada fator, sendo a meia vida mais longa a do fator II, dessa forma é justificado a conduta clínica para a não troca da varfarina antes de 5 dias do início do tratamento, para que se espere o efeito de anticoagulação máxima ser atingida; e para o uso simultâneo de anticoagulantes de ação mais rápida, que seriam as heparinas, por pelo menos 5 dias (Zago et al., 2013).

No mercado brasileiro existem disponíveis dois tipos de heparinas, a HNF e a HBPM, entre as quais se destaca no uso hospitalar a Enoxaparina, que é um tipo de HBPM, com aprovação para os seguintes tratamento: síndromes coronárias agudas, tratamento e profilaxia de TVP e tratamento para EP, e para prevenção de formação de trombos circulantes na circulação extracorpórea durante o processo de hemodiálise (ISMP, 2020; Brasil, 2020d; Jupalli \& lqbal, 2021).

A prevenção da trombose venosa é eficaz, segura e individualizada para cada paciente. Após detectar a classificação de risco dos pacientes na unidade hospitalar, (enfermarias ou em UTI's - Unidade de terapia intensiva), são tomadas medidas preventivas como o incentivo à deambulação, uso de meia elásticas compressivas, membros inferiores elevados objetivando aumentar o fluxo sanguíneo e assim, otimizar a microcirculação, além do uso racional de anticoagulantes. Os pacientes cirúrgicos são classificados de acordo com o escore de Caprini, sendo inferior a 3. Porém, neste escore pode haver mudanças conforme a necessidade clínica do paciente. Recomenda-se que pacientes com risco inferior a 5 recebam 5.000 UI de heparina não fracionada subcutânea (SC) ou $20 \mathrm{mg}$ de Enoxaparina por via SC a cada 12/12 horas. Para pacientes com alto risco, é recomendado o uso de 5.000UI de heparina não fracionada de 8/8 horas ou Enoxaparina $40 \mathrm{mg}$ por via SC 1x/dia (Scaravonatti et al, 2021).

Em comparação com a HNF, o uso da enoxaparina apresenta vantagens, como administração mais fácil e resposta posológica mais confiável, além de estar associada com um menor número de osteopenia e de trombocitopenia, porém é considerada uma droga potencialmente perigosa por estar relacionada a erros de medicação, como no caso de dosagem incorreta. Outra vantagem, é que a HBPM possui capacidade reduzida de ligação com as proteínas plasmáticas, tendo em vista o tamanho menor da molécula, aumentando assim sua biodisponibilidade. A dose de enoxaparina deve ser consistente com suas indicações de tratamento e ajustada conforme o peso corporal do paciente e a depuração da creatinina. É necessário monitorar as doses de longo prazo (> 7 a 10 dias) em pacientes com insuficiência renal; disfunção hepática; pacientes com sobrepeso; mulheres grávidas; recém-nascidos; crianças; e idosos. O monitoramento por dose do fator Xa é suficiente para 
viabilizar o uso seguro, porém para pacientes estáveis e sem complicações, não se faz necessário o monitoramento de rotina, devido ao perfil farmacodinâmico previsível (Brasil, 2020d; Zago et al., 2013).

Devido a variabilidade da resposta à dose e às mudanças na resposta do paciente ao longo do tempo, a administração de HNF requer monitoramento e ajustes de dose. Como os níveis plasmáticos de HNF não podem ser medidos diretamente, a resposta ao anticoagulante administrado pela via EV é monitorada pela ativação do tempo de tromboplastina parcial (ATTP), que é a unidade de medida do tempo em segundos da formação de trombo. Ao início da terapia é recomendado a realização da ATTP e após 6 horas do início da infusão, sendo necessário a realização do exame a cada 6 horas. As complicações principais causadas pela terapia com HNF está o sangramento, trombocitopenia, osteoporose. Sendo os fatores de riscos importantes para determinar os riscos de sangramento, a idade, sexo, função renal, peso corporal, cirurgias recentes e o consumo de álcool (Nutescu et al., 2016).

A iniciação rápida de heparina se dar pela via $\mathrm{EV}$, porém a SC também pode ser uma via de administração para tratamento agudo, porém as injeções SC devem ter ajuste para doses mais altas, devido a biodisponibilidades das heparinas, no caso da HNF a biodisponibilidade varia de 30 a 70\%, mas para a disponibilidade das HBPM após administração por via SC se aproximam de $100 \%$. Comparando a heparina EV com a heparina SC ajustada por dose para o tratamento inicial de TVP, a heparina SC tem um risco menor de recorrência e extensão de TEV, e um risco equivalente de sangramento maior (Smythe et al., 2016; Nutescu et al., 2016).

Já os antagonistas da vitamina $\mathrm{K}$, requerem dose individual em razão aos altos números de fatores que podem afetam sua farmacologia, fatores esses intrínsecos ou externos, como: o metabolismo, que podem variar de paciente a paciente, além das possíveis alterações metabólicas sofridas no processo de absorção, eliminação e metabolismo hepático; a dieta do paciente que dependendo do teor de vitamina $\mathrm{k}$ modificam o efeito dos antagonistas; além disso doenças que afetam o fígado e a função sanguínea, como hepatite aguda ou crônica e insuficiência cardíaca. Sendo a eficácia e segurança da utilização dos antagonistas de vitamina $\mathrm{K}$ associados fortemente com o controle (Gómez-Rosas et al., 2021; Ceresetto et al., 2020).

A idade também é outro fator que pode interferir nos efeitos dos antagonistas da vitamina $\mathrm{k}$, pois para os idosos as dosagens dos antagonistas precisam ser menores, devido à diminuição da concentração sérica de albumina e diminuição do metabolismo hepático, resultando em uma disponibilidade do fármaco aumenta (Ceressetto et al., 2020). Não é recomendado de varfarina, esse que é o principal representante dos antagonistas da vitamina K, pois atravessam a barreira placentária, fazendo com que os níveis plasmáticos fetais sejam semelhantes aos valores maternos, o que pode causar sangramento no feto. Além disso o uso da varfarina durante a gravidez geralmente leva a aborto, natimorto, parto prematuro e morte neonatal. Os benefícios e riscos da terapia com varfarina devem ser avaliados cuidadosamente, devendo ser evitados durante o primeiro trimestre e próximo ao parto (Patel et al., 2021).

Diante disso, a participação do farmacêutico no âmbito hospitalar para a profilaxia medicamentosa voltada para o TEV vem sendo consolidada e obrigatória nos programas de educação hospitalares, pois mostra que o uso de medidas preventivas de TEV aumentou de $43 \%$ para 58\%, tendo bons resultados alcançados na prevenção pela intervenção de farmacêuticos clínicos, devido a atuação em visitas multiprofissionais, ao identificar falhas no monitoramento, erros nas dosagens prescritas, falhas no ajuste de dose e falhas nas prescrições voltadas às gestantes, pacientes com insuficiência renal crônica e pacientes com risco aumentado de sangramento (Haga et al., 2014; ISMP, 2020).

Sendo importante que o hospital desenvolva uma estratégia para reduzir as chances de TEV em pacientes hospitalizados, por exemplo trabalhar com base em protocolos já estabelecidos (Ex. protocolo de TEV). Sem uma direção de trabalho, as consequências podem ser fatais, incluindo o aumento do número de dias de hospitalização e a evolução do quadro para uma possível evolução de EP. A implementação dos serviços de farmacêuticos clínicos nas áreas verticais incluem prevenção de TEV e a promoção de saúde. As intervenções realizadas pelo papel do farmacêutico clínico são de extrema 
importância, sendo eles: prevenção de drogas, combinação de dose e posologia, ajuste da via de administração, reposição preventiva de medicamentos e descontinuação preventiva para evitar a repetição do tratamento.

Diante disso, é importante enfatizar medidas preventivas, estimulando o sistema de saúde a desenvolver estratégias para garantir as melhores práticas de prevenção agregando recursos suficientes a essas ações para que os hospitais possam desenvolver novas estratégias de estratificação de risco para a aplicação correta e prevenção de trombose venosa profunda em ambiente hospitalar (Scaravonatti et al., 2021).

\section{Considerações Finais}

Com base nos estudos, pode ser observado que a reação adversa comum entre os anticoagulantes citados é a presença de possíveis casos de hemorragias, sendo que atualmente o anticoagulante mais utilizado e que está preconizado pelo Ministério da Saúde dentro do ambiente hospitalar é a Enoxaparina classificada como HBPM, por apresentar vantagens em relação a HNF como administração mais fácil e resposta posológica mais confiável, além de estar associada com um menor número de osteopenia e de trombocitopenia.

Apesar dos antagonistas de vitamina $\mathrm{K}$ serem efetivos no tratamento da TVP, essa classe de anticoagulantes apresenta o risco maior de sangramento, além de interagir com medicamentos, alimentos e não serem recomendados para pacientes grávidas. Já no idoso é necessário o ajuste de dose, em decorrência das alterações farmacocinéticos do envelhecimento. Seguindo na classe de antagonistas da vitamina K, a Varfarina tem ação e eliminação mais demorada, apresentando eficácia no tratamento a longo prazo.

Diante do exposto, conclui-se a necessidade da presença do farmacêutico clínico/hospitalar junto a equipe multidisciplinar para a promoção de saúde, garantindo o acesso e a qualidade da terapêutica farmacológica com base na TVP em membros inferiores de pacientes hospitalizados. Fica claro que sua participação neste processo profissional e educativo se inicia no momento da internação havendo uma comunicação entre as equipes multidisciplinares para uma terapêutica adequada a cada tipo de caso, dando continuidade com o elo até a alta hospitalar, fase pelo qual o paciente é orientando sobre a profilaxia, combinação de dose e posologia, ajuste da via de administração e interações alimentar e medicamentosas.

\section{Referências}

Alves, C. P., Almeida, C.C. \& Balhau, A. P. (2015) Tromboembolismo venosa diagnóstico e tratamento. Lisboa: Sociedade Portuguesa de Cirurgia. https:// https://www.spcir.com/wp-content/uploads/2016/06/Tromboembolismo_Venoso_Diagnostico_e_Tratamento_2015.pdf

Brandão, G. M. S., Cândido, R. C. F., Rollo, H. de A., Sobreira, M. L. \& Junqueira, D. R. (2018). Anticoagulantes orais diretos para o tratamento da trombose venosa profunda: revisão de revisões sistemáticas. Jornal Vascular Brasileiro, 17(4), 310-317.

Brasil. Ministério da Saúde. (2016). Saiba como evitar a trombose. https://www.brasil.gov.br/saude/2016/09/saiba-como-evitar-a-trombose

Brasil. Ministério da Educação. (2020a). 16 de setembro: Dia Nacional de Combate e Prevenção á Trombose. https://www.gov.br/ebserh/pt-br/hospitaisuniversitarios/regiao-centro-oeste/hc-ufg/comunicação/noticias/16-de-setembro-dia-nacional-de-combate-e-prevencao-a-trombose

Brasil. Governo do Brasil. (2020b). Tratamento de Trombose Venosa Profunda. https://www.gov.br/pt-br/servicos-estaduais/tratamento-de-trombose-venosaprofunda-1\#outras

Brasil. Ministério da Saúde. (2020c). Informe diário de evidencias | COVID-19. N35. https://www.gov.saude/pt-br/coronavirus/publicaçoestecnicas/evidencias-cientificas/n35-22-05-2020-informe-diario/view

Brasil. Ministério da Saúde. (2020d). Portaria conjunta $N^{\circ} 04$, de 12 de fevereiro de 2020. https://www.in.gov.br/web/dou/-/portaria-conjunta-n-4-de-12-defevereiro-de-2020-243406512

Brunton,L. L., Hilal-Danda, R. \& Knollmann, B. C. (2019). As bases Farmacológicas da Terapêutica de Goodman e Gilman. (13. Ed.) Porto Alegre: AMGH.

Cagnolati, D., Sankarankutty, A. K., Rocha, J. P. S., Beer, A. \& Silva, O. C. e. (2017). Hemostasia e distúrbios da coagulação. Faculdade de Medicina de Ribeirão Preto - USP/SP. https://sites.usp.br/dcdrp/wp-content/uploads/sites/273/2017/05/hemostasia_revisado.pdf

Ceressetto, J. M., Duboscq, C., Korin, J., Fondevila, C., Casais, P., Rossi, A., Scazziota, A., Martinuzzo, M., Cancela, M. E. A., Rosa, C., Pombo, G., Guimelli, C., Adamczuk, Y., Martí, A., Penchasky, D., Riveros, D., Puente, D., celebrin, L., Molnar, S., Meschengieser, S., Ríos, m. C., Blanco, A., Hendler, 
H., Brodsky, A., Luceros, A. S., Aranguren, P. N., Fedele, j. L., Merlo, C., Raña, P., Gumpel, C., Colorio, C., Grand, B., Canónico, v., Rossi, E., Colimodio, P., Orlando, S., Fassi, D., Arias, M., Viudez, L., Farreras, R., Martínez, P., Ferro, H., Casali, C., Baques, A., Arbesú, G., Viñuales, S., Medina, F., Cortés, V., Gallo, M. D. C., Ehelou, L., Barrera, L., Rey, I., Larrañaga, G., Figueroa, F., Vilaseca, A., Kuri, I., Maneyro, A., Otaran, M., Xavier, D. L., Garbiero, S., Salviú, J., fontenla, P., Eckhardt, A., Bomparola, C. \& Fornasiero. (2020). Consenso argentino em gestión efectiva de clinicas de anticoagulación para uso de antagonistas de la vitamina K. Medicina (Buenos Aires), 80(4), 1-26.

Farhat, F. C. L. G., Gregório, H. C. T. \& Carvalho, R. D. P. (2018). Avaliação da profilaxia da trombose venosa profunda em um hospital geral. Jornal Vascular Brasileiro, 17(3) 184-192.

Fuzinatto, F., Wajner,A., Waldemar, F. S. de, Hopf, J. L. de S., Schuc, J. F. \& Barreto, S. S. M. (2011). Profilaxia para tromboembolia venosa em um hospital geral. Jornal Brasileiro de Pneumologia, 37(2), 160-167.

Gómez-Rosas, P., Sosa-Velázquez, M. Á., Yáñez-Funes, L. A., González-Trejo, J. J., Majluf-Cruz, K., Isordia-Salas, I., Ignacio-Ibarra, G., Almaraz-Delgado, M., Moreno-Ruiz, L. A. \& Valencia-Chávez, J. S. (2021). Analysis of the quality of anticoagulation with vitamin K antagonista in three clinical scenarios in Mexico. Gaceta de Mexico, 157(3), 284-292.

Harter, K., Levine, M. \& Henderson, S. (2015). Anticoagulation Drug therapy: a review. Western Journal Of Emergency Medicine, 16(1), 11-17.

Haga C. S., Mancio, C. M., Pioner, M. C., Alves, F. A., Lira, A. R., Silva, J. S., Ferracini, F. T., Borges Filho, W. M., Guerra, J. C. \& Laselva, C. R. (2014). Implementation of vertical clinical pharmacist servisse on venous thromboembolism prophylaxis in hospitalized medical patients. Einstein (São Paulo), 12(1), 27-30.

ISMP. Instituto para práticas Seguras no Uso de Medicamentos. (2020). Enoxaparina: erros de medicação, riscos e práticas seguras na utilização. Boletim ISMP. 9(4), 1-9. https://www.ismp-brasil-org/site/wp-content/uploads/2020/07/BOLETIM_ISMP_JUNHO_ENOXAPARINA.pdf

Jupalli, A. \& lqbal, M. A. (2021). Enoxaparin. StatPearls [Internet]. https://www.ncbi.nlm.nih.gov/books/NBK539865

Katzung, B. G., Masters, S. B. \& Trevor, A. J. (2014). Farmacologia Básica e clínica. (12. ed). Porto Alegre.

Mendonça, M. M. V., Santos, A. C. de F., Mendes, A. C. R., Guimarães, B. A., Ribeiro, G. A., Andrade, I. A. de, Potsch, L. S., Jesus, L. S. de, Nicolau, L. F. M. \& Parreira, M. L. B. Q. C. (2021). A incidência de tromboembolismo venoso em gestantes e no puerpério e seus fatores de risco. Revista Eletrônica Acervo Cientifico, 30, 1-6. DOI: 10.25248/REAC.e8125.2021

Mioc, M. L., Prejbeanu, R., Vermesan, D., Haragus, H., Niculescu, M., Pop, D. L., Balanescu, A. D., Malita, D. \& Deleanu, B. (2018). Deep vein thrombosis following the treatment of lower limb pathologic bone fractures - a comparative study. BMC musculoskeletal disordes, 19(1), 1-5.

Nutescu, E. A., Burnett, A., Fanikos, J., Spinler, S. \& Wittkowsky, A. Pharmacology of anticoagulants used in the treatment of venous thromboembolism. Journal of Thrombosis and Thrombolysis, 41(1), 15-31.

Ohki, A. V. \& Van Bellen, B. (2017). A incidencia regional do tromboembolismo venoso no Brasil. Jornal Vascular Brasileiro, 16(3), $227-231$.

Okuhara, A., Navarro, T. P., Procópio, R. J. \& Leite, J. O. M. de. (2015). Incidência de trombose venosa profunda e estratificação dos grupos de risco em serviço de cirurgia vascular de hospital universitário. Jornal Vascular Brasileiro, 14(2), 139-144.

Patel, S., Singh, R., Preuss, C. V. \& Patel, N. (2021). Warfarin. StatPearls [Internet]. https://www.ncbi.nlm.nih.gov/books/NBK470313/

Pereira, M. A. de M., Gomes, M. G. P., Madureira, N. A. B., Bersan, P. N., Silva, R. T., Vilela, R. V., Silva, S. R. da, Martins, T. S. A. \& Krettli, W. S. da C. (2011). Diagnóstico da trombose venosa profunda e particularidades na gravides e puerpério. Revista Médica de Minas Gerais, 21(4-6), 11-14.

Rang, H. P., Ritter, J. M., Flowerr, R. J. \& Henderson, G. (2016). Rang \& Dale: Farmacologia. (8. Ed.). Rio de Janeiro: Elsevier.

Raphael, A. (2020). Moving towards ideal and appropriate models of anticoagulation management service. Annals Of African Medicine, 19 (3), 153 - 163.

Rodrigues, E. S., Castilho-Fernandes, A., Covas, D. T. \& Fontes, A. M. (2012). Novos conceitos sobre a fisiologia da hemostasia. Revista da Universidade Vale do Rio Verde, Três Corações, 10(1), 218-233.

Scaravonatti, M. E., Scaravonatti, M., Kawai, A. \& Linartevichi, V. (2021). Aplicação de profilaxia da trombose venosa profunda em unidade de terapia intensiva. Fag Journal of Health (FJH), 3(2), 129-139. https://doi.org/10.35984/fjh.v3i2.328

Shen, M., Cutreta, N. J., Dodd, A. C., Wallase, C., Avilucea, F. R., Melbourne, C., Jahangir, A. A., Mir, H. H., Obremskey, W. T. \& Sethi, K. M. (2017). The risk of deep vein thrombosis in total joint patients compared to orthopaedc trauma patients:Need for new preventoin guidelines. Journal of Clinical orthopaedics and trauma, 8(2), 52-56.

Silva, R. D. R \& Melo, E. M. (2016). A atual teoria da coagulação baseada em superfície celulares. Revista Acadêmica do Instituto de Ciências da Saúde, 2(1), $79-82$.

Smythe, M. A., Priziola, J., Dobesh, P. P., Wirth, D., Cuker, A. \& Wittkowsky. (2016). Guidance for the practical management of the heparina anticoagulants in the treatment of venous thromboembolism. Journal of Thrombosis and Thrombolysis, 41(1), 165-186.

Sousa, I. C. A. \& Álvares, A. C. M. (2018). A trombose venosa profunda como reação adversa do uso contínuo de anticoncepcionais orais. Revista de divulgação Científica Sena Aires. 7(1), 54-65.

Wolberg, A. S., Aleman, M. M., Leiderman, K. \& Machlus, K. R. (2012). Procoagulant Activity in Hemostasis and Thrombosis. Anesthesia \& Analgesias, 114(2), 275-285.

Yoshida, W. B. (2016). Tratamento convencional da trombose venosa profunda proximal: ainda uma boa opção?. Jornal Vascular Brasileiro, 15(1), 1-3.

Zago, M. A., Falcão, R. P., Pasquini, R., Spector, N. V., Cavas, D. T. \& Rego, E. M. (2013). Tratado de Hematologia. Editora Atheneu. 